\title{
Current status of maintenance therapy for advanced ovarian cancer
}

This article was published in the following Dove Press journal:

International Journal of Women's Health

29 October 2009

Number of times this article has been viewed

\section{Joanie Mayer Hope \\ Stephanie V Blank}

New York University School of Medicine, Division of Gynecologic Oncology, New York NY, USA
Correspondence: Stephanie V Blank New York University School of Medicine, Division of Gynecologic Oncology, 160 East 34th Street, New York, NY, USA

Email stephanie.blank@nyumc.org
Abstract: Even after countered with and responding to maximal surgical and chemotherapy efforts, advanced ovarian cancer usually ultimately recurs. One strategy employed to forestall recurrence is maintenance chemotherapy, an extension of treatment following a complete response to conventional measures. Many agents have been studied and many more are currently under investigation in maintenance regimens. While phase III data suggest that taxane maintenance prolongs progression-free survival, no overall survival benefit has been established. This article reviews the current status of maintenance therapy for advanced ovarian cancer, including phase III evidence and new and upcoming trials.

Keywords: maintenance therapy, consolidation therapy, advanced ovarian cancer

\section{Introduction}

Ovarian cancer continues to be associated with very high morbidity and mortality. In 2009, there will be an estimated 21,550 new cases of ovarian cancer diagnosed in the United States and 14,600 deaths. ${ }^{1}$ Even with optimal frontline treatment involving aggressive surgical cytoreduction followed by platinum and taxane-based chemotherapy, 5-year survival for women with advanced stage disease is only $45 \%$. Furthermore, over $50 \%$ of women who achieve a complete response to initial treatment will relapse within 18 to 24 months. ${ }^{2}$ While effective second-line treatments are available, response rates drop with each subsequent recurrence due to the onset of drug resistance. From this background, the notion of extended chemotherapy following complete response to conventional treatment has been developed in an effort to delay or even avoid recurrence completely.

\section{Definitions}

Two basic approaches have been taken to extended chemotherapy: 1) consolidation or intensification therapy, and 2) maintenance chemotherapy. These terms are indistinct and often confused in the literature. Consolidation therapy generally involves the addition of an intense short-term treatment immediately following the completion of front-line therapy. Whole abdominal radiation, radioimmunotherapy, intraperitoneal chromic phosphate $\left.{ }^{32} \mathrm{P}\right)$, and high-dose cytotoxic chemotherapy with stem cell support have all been studied with little proven benefit but with substantial toxicity for ovarian cancer patients (see Table 1). ${ }^{3-18}$ Maintenance chemotherapy, on the other hand, involves lower-dose treatments over a more prolonged period following a clinical remission from a standard regimen. The data 


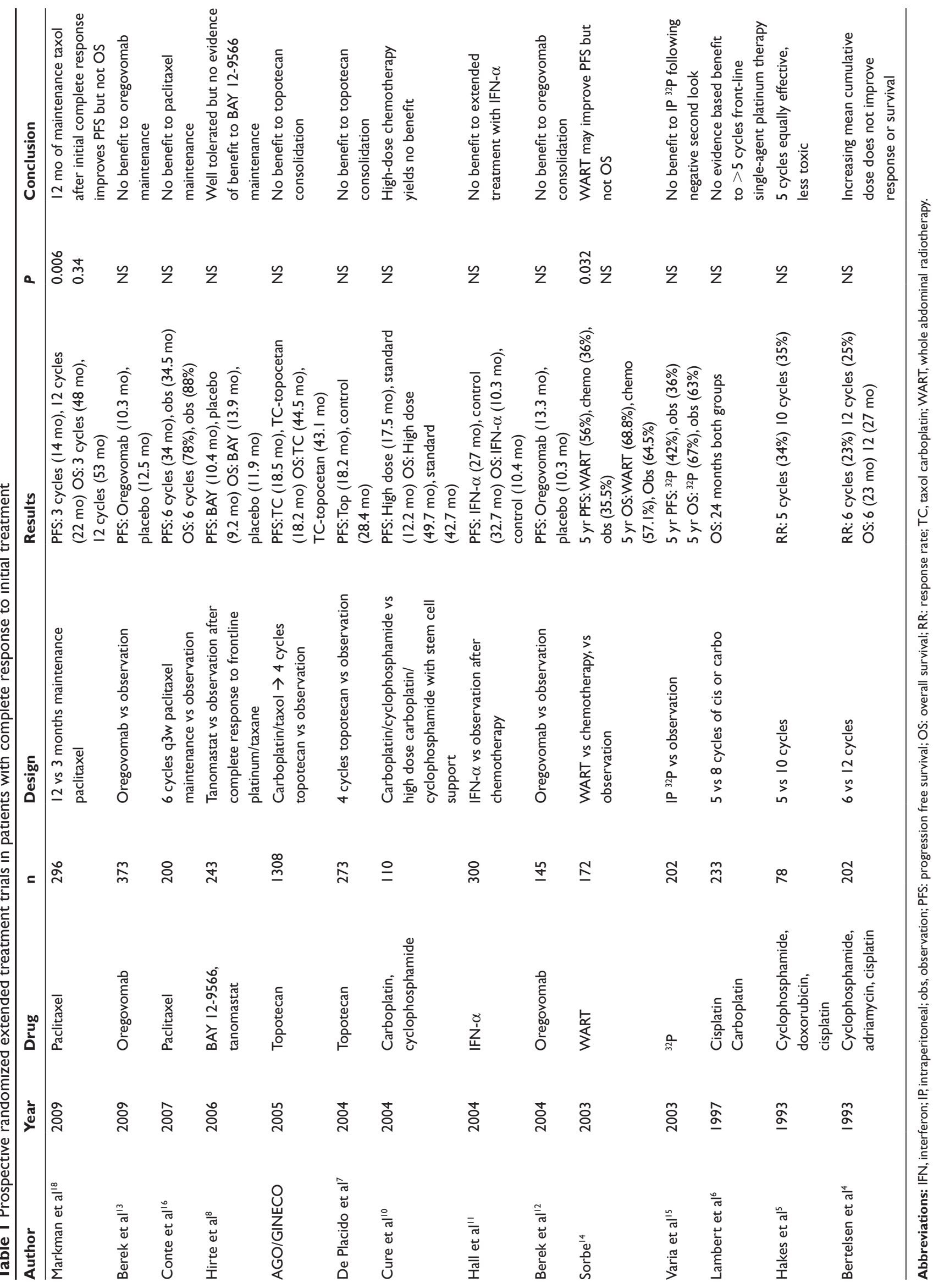


here are more promising, and a review of the phase III evidence is the subject of this paper.

\section{Rationale}

The rationale supporting maintenance chemotherapy is based on the theory that slowly dividing tumor cells which were inadequately exposed to front-line cycle-dependent cytotoxic treatment may be effectively eliminated by continued treatment over time. ${ }^{19,20}$ In addition to targeting the remaining tumor burden, prolonged chemotherapy with known antiangiogenic agents may forestall new tumor growth. Opponents of maintenance therapy argue that resting after primary chemotherapy allows for recovery from the toxic effects, and that waiting for recurrence increases the likelihood of repopulation with chemo-responsive cells. These arguments are founded in the concern that maintenance treatment may obviate the benefit of retreatment when relapse occurs. ${ }^{6}$ Randomized controlled data have yet to resolve this debate definitively. Additionally, the effect of maintenance therapy on quality of life needs to be considered when making decisions about continuing or stopping treatment once complete response is reached.

\section{Phase III clinical trials: a historical perspective}

In the 1990s, several randomized trials addressed the question of whether extending the number of platinum cycles during front-line chemotherapy would benefit survival. Eight, 10 , and 12 cycles were compared to the standard of 5 or 6 , and no improvement in response or prolongation of survival was established. ${ }^{4-6}$ Patients in these trials were randomized prior to initiation of front-line treatment rather than after determination of clinical response. Therefore, platinumresistant patients (roughly $25 \%$ of women with ovarian cancer) were randomized to receive more of a drug to which they were probably not responding in the first place. Furthermore, the cumulative toxicity of extended platinum therapy made it a questionable choice as a maintenance agent.

Many subsequent trials have sought to avoid similar design flaws by establishing documented complete clinical response as inclusion criteria. However, phase III trials of extended treatment with topotecan, whole-abdominal radiation, intraperitoneal ${ }^{32} \mathrm{P}$, high-dose cytotoxic regimens, antiangiogenic matrix metalloproteinase inhibitors, and immunotherapies such as interferon alpha have all failed to demonstrate survival advantages (Table 1)., ${ }^{3,7-16}$ The only positive randomized controlled data to date involve the use of paclitaxel. Taxanes are compelling as maintenance agents because in addition to being cytotoxic, they have antiangiogenic activity which may be enhanced by prolonged and effectively spaced treatments. ${ }^{17,18}$

In 2003, Markman et al published initial results from the SWOG S9761/GOG 178 collaborative trial in which advanced stage ovarian cancer patients with complete clinical response to platinum/taxane therapy were randomized to receive either 3 or 12 cycles of monthly paclitaxel (175 mg/m $\mathrm{m}^{2}$ in a 3 -hour infusion). ${ }^{17}$ At the interim analysis, $34 / 112$ patients in the 3 -cycle arm had relapsed, compared to $20 / 110$ patients in the 12 -cycle arm $(P=0.0023)$, translating to a median progression-free survival advantage of 7 months (21 vs 28 months). The SWOG data safety and monitoring committee discontinued the trial on basis of a prespecified termination boundary of $P=0.005$. At the time the study was closed, no significant overall survival advantage was demonstrable. ${ }^{17}$

In 2009, mature results from GOG 178 were published, confirming an 8-month progression-free survival advantage in the 12-cycle arm ( 22 vs 14 months, $P=0.006$ ), but failing to establish a overall survival advantage (53 vs 48 months, $P=0.34) .{ }^{18}$ The authors hypothesized that a potential survival advantage may have been obviated by 1) insufficient sample size, 2) crossover patients in the 3-cycle arm who actually received more cycles ( $6 \%$, or 9 patients), or 3 ) the equalizing effects of treatments initiated once relapse occurred. Of note, a second randomized trial of paclitaxel maintenance conducted by Conte et al failed to show either progression-free survival or overall survival benefit. In this trial, 200 advanced ovarian cancer patients with complete response to platinum/paclitaxel treatment were randomized to single-agent paclitaxel every 3 weeks for 6 cycles versus observation. At 44 months, median progression-free survival and 3-year overall survival were 34 months and $88 \%$, respectively, in the observation arm, compared to 34.5 months and $78 \%$ in the paclitaxel arm. ${ }^{16}$

\section{Ongoing randomized controlled trials}

Several ongoing phase III clinical trials have been designed to determine whether maintenance chemotherapy confers a survival advantage in ovarian cancer patients (see Table 2). The taxane question will be addressed directly by GOG 212 - a 3-arm randomized trial of maintenance chemotherapy comparing 12 months of single-agent paclitaxel to polyglutamate paclitaxel (Xyotax ${ }^{\mathrm{TM}}$, or PPX) or observation alone until documented relapse in stage III or IV ovarian epithelial ovarian or peritoneal cancers. PPX is a drug conjugate which links poly-L-glutamic acid, a biodegradable 


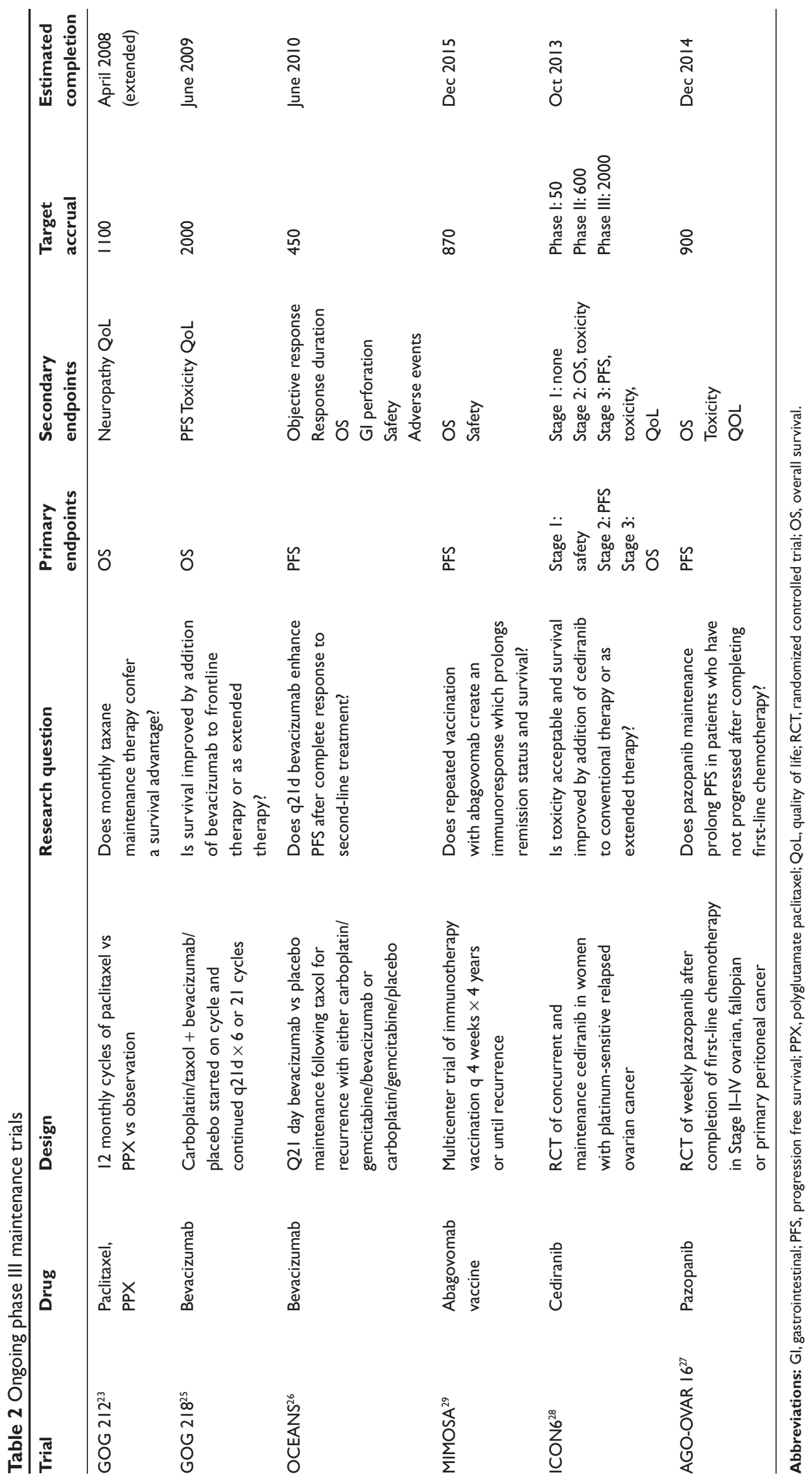


polymer, to paclitaxel. The conjugate confers molecular stability within the systemic circulation and enhances passive accumulation in tumor tissue where PPX progressively releases its active taxane constituent. ${ }^{22}$ Eligibility includes optimally surgically cyto-reduced patients $(\leq 1 \mathrm{~cm}$ of residual disease) who have had a complete response to adjuvant platinum/taxane treatment as well as patients who have received neoadjuvant chemotherapy followed by surgery to no residual disease. Primary outcome of this trial is overall survival. Secondary outcomes include quality of life, peripheral neuropathy, and a series of exploratory angiogenic markers. ${ }^{23}$

The use of biologic agents for maintenance therapy in both front-line and recurrent settings is currently the focus of avid ovarian cancer research. Bevacizumab is a humanized recombinant monoclonal antibody, which targets vascular endothelial growth factor (VEGF), an important factor in tumor angiogenesis. Preclinically, mouse xenograft models have demonstrated that bevacizumab inhibits recurrence and prolongs survival when given as maintenance therapy 3 weeks after induction combination chemotherapy. ${ }^{24}$ Bevacizumab maintenance is addressed in GOG 218, a randomized controlled trial in which advanced ovarian, primary peritoneal, and fallopian tube cancer patients with measurable disease are treated with frontline carboplatin and pacliaxel. Bevacizumub or placebo is then added on cycle 2 and continued every 21 days for either 6 or 22 cycles. The primary endpoint is overall survival and secondary endpoints include progression-free survival, toxicity and quality of life. GOG 218 completed enrollment in June 2009, having achieved target accrual of 2000 patients. $^{25}$

Bevacizumab is also being investigated as maintenance after complete response to second-line treatment. The OCEANS trial is 2-part placebo-controlled, randomized, multicenter, industry-sponsored phase III study which compares bevacizumab in combination with carboplatin/ gemcitabine to the same regimen with placebo in women with platinum-sensitive recurrent epithelial ovarian, primary peritoneal, or fallopian tube carcinoma. ${ }^{26}$ In the OCEANS protocol, trial participants who demonstrate complete response to carboplatin/gemcitabine plus bevacizumab or placebo are then offered maintenance treatments with bevacizumab or placebo every 3 weeks for 1 year.

Other potent inhibitors of VEGF receptor tyrosine kinases are also under phase III investigation. In the frontline setting is pazopanib, a small-molecule inhibitor of cKit which targets platelet derived growth factor receptor as well as VEGF receptors 1, 2, and 3. In the AGO-OVAR 16 trial, women who have not progressed after first-line chemotherapy for epithelial ovarian, fallopian tube, or primary peritoneal cancer are randomized to either pazopanib or placebo $800 \mathrm{mg}$ daily for 52 weeks (12 months). The accrual goal is 900 with primary endpoint of progression-free survival and secondary endpoints including overall survival, toxicity, and quality of life. ${ }^{27}$

In the setting or recurrence, the drug cediranib will soon be under investigation in the ICON6 trial. Cediranib (also know as AZD2171) is a once-daily oral therapy which targets VEGF 1, 2, and 3 and competes with adenosine triphosphate. ICON6 is a multicenter-multiphase trial in which patients with recurrent ovarian cancer will be randomized to A) carboplatin, paclitaxel and placebo for 6 cycles followed by placebo maintenance for 18 months; B) carboplatin, paclitaxel and cediranib with placebo maintenance; or C) carboplatin, paclitaxel and cediranib followed by cediranib maintenance. The trial incorporates a phase I, II, and III component with accrual goals of 50, 600, and 2000 for each phase, respectively. ${ }^{28}$

Another innovative approach to maintenance involves the concept of immunotherapy. The MIMOSA trial (Monoclonal antibody Immunotherapy for Malignancies of Ovary by Subcutaneous Abagovomab) is a phase III trial involving the administration of an antibody which functionally mimics the CA125 antigen and induces humoral and cellular CA125specific immunity. ${ }^{29}$ The trial involves repeated vaccination every 4 weeks for up to 4 years or until recurrence in ovarian cancer patients with complete clinical response to frontline treatment. MIMOSA has now completed accrual and results are anticipated.

Of note, previously published phase III data on the related drug oregovamab (a monoclonal antibody to CA125) have not demonstrated benefit as either a maintenance or a consolidation agent. Berek et al investigated the role of maintenance mono-immunotherapy with oregovomab in a placebo-controlled blinded trial. Drug or placebo was given to ovarian cancer patients after complete clinical response from front-line therapy every 4 weeks for 3 cycles and then every 12 weeks for 5 years or until recurrence. In a 2:1 randomization, 251 patients were given drug and 100 given placebo, without difference in clinical outcome. A relatively modest immune response was seen in participants compared to the same drug given in front-line or in recurrent settings in combination with other chemotherapies. The authors postulated that the maintenance setting may not be the most effective time to administer immunotherapy, given the 


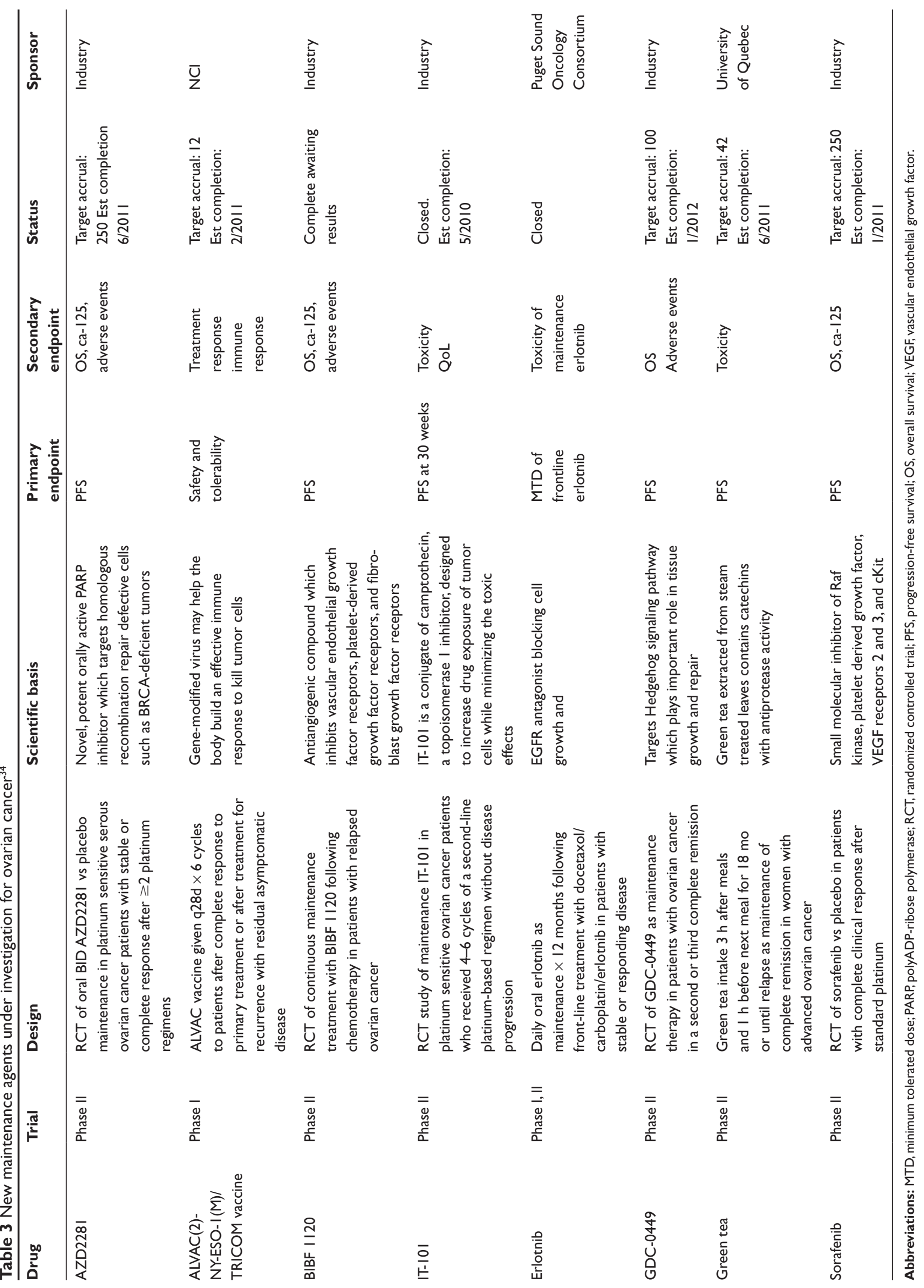


relatively low tumor burden and hence minimal circulating tumor antigen targeted by this approach. ${ }^{12,13}$

\section{Toxicity and quality of life considerations}

When considering whether to give extended and potentially morbid treatments to women in clinical remission, the question of quality of life (QoL) must be addressed. Without clear evidence of survival advantage, causing patients to potentially feel sicker during times of clinical remission is of utmost concern. Unfortunately, data addressing the impact of maintenance chemotherapy on QoL are currently lacking. GOG 178 did not include a QoL component, leaving unanswered questions about treatment-associated neurotoxicity from prolonged taxane exposure. Markman reported major differences in treatment-related sensory neuropathy between the 3- and 12-cycle arms of GoG 178: $35 / 149$ grade 2 events and 9/149 grade 3 events in the 12-cycle arm compared with 20/136 grade 2 and 1/136 grade 3 events in the 3 -cycle arm. The trial did not prospectively evaluate the duration of neuropathy or the persistence of symptoms after discontinuation of treatment. ${ }^{18}$

Robinson et al also addressed the taxane question in a phase II feasibility trial of paclitaxel maintenance therapy following front-line intravenous/intraperitoneal (iv/ip) cisplatin/paclitaxel versus iv carboplatin/paclitaxel. Robinson found that completion rates of the maintenance portion of the regimen were higher in the iv/ip group. Patients who stopped maintenance therapy usually stopped early (within 3 cycles) and the most common reasons in descending order included neuropathy, fatigue, myelosuppression, and disease progression. ${ }^{30}$ The ongoing GOG 212 does include QoL as a secondary endpoint. In addition, a variety of novel taxanes and taxane-like compounds are under investigation which may have more acceptable toxicity profiles as maintenance agents. ${ }^{22,31-33}$

\section{Into the future}

Looking beyond phase III data, the notion of extended chemotherapy treatment is an active area of research extending far beyond the realm of traditional cytotoxic regimens. In a host of ongoing phase I and II trials, polyADP-ribose polymerase (PARP) inhibitors, immuno-vaccinations, endothelial and epithelial growth factor inhibitors, novel VEGF receptor inhibitors, and even concentrated green tea are being explored for their potential maintenance benefits (see Table 3). ${ }^{34}$ For patients who want to take proactive therapeutic measures to delay recurrence, these maintenance trials may offer an opportunity to meet this need. For clinicians, these trials represent cutting edge scientific efforts to tackle one of the most significant problems in ovarian cancer treatment - prevention of recurrence.

\section{Conclusion}

The question of the utility of maintenance chemotherapy in ovarian cancer has not been answered. Yet there is promising research underway and patients should be encouraged to participate. Within the coming years, a host of novel more targeted and less toxic therapies can be expected to enter the line-up for phase III maintenance therapy interrogation.

\section{Disclosures}

The authors have no conflicts of interest to disclose.

\section{References}

1. National Cancer Institute Cancer Stat Fact Sheets: http://seer.cancer gove/csr/1975_2006/results_single/sect_01_table.01.pdf

2. Cancer Facts and Figures 2008, American Cancer Society. www.cancer. org/docroot/stt/content/stt_1x_cancer_facts_and_figures_2008.asp

3. Gadducci A, Cosio S, Conte PF, Genazzani AR. Consolidation and maintenance treatments for patients with advanced epithelial ovarian cancer in complete response after first line chemotherapy: a review of the literature. Crit Rev Oncol Hematol. 2005;55:153-166.

4. Bertelsen K, Jakobsen A, Strøyer J, et al; A prospective randomized comparison of 6 and 12 cycles of cyclophosphamide, adriamycin, and cisplatin in advanced ovarian cancer: a Danish Ovarian Study Group trial (DACOVA). Gynecol Oncol. 1993;49(1):30-36.

5. Hakes TB, Chalas E, Hoskins WJ, et al. Randomized prospective trial of 5 versus 10 cycles of cyclophosphamide, doxorubicin, and cisplatin in advanced ovarian carcinoma. Gynecol Oncol. 1992;45(3):284-289.

6. Lambert HE, Rustin GJ, Gregory WM, Nelstrop AE. A randomized trial of five versus eight courses of cisplatin or carboplatin in advanced epithelial ovarian carcinoma. A North Thames Ovary Group Study. Ann Oncol. 1997;8(4):327-333.

7. De Placido S, Scambia G, Di Vagno G, et al. Topotecan compared with no therapy after response to surgery and carboplatin/paclitaxel in patients with ovarian cancer: Multicenter Italian Trials in Ovarian Cancer (MITO-1) randomized study. J Clin Oncol. 2004;22(13): 2635-2642.

8. Hirte H, Vergote IB, Jeffrey JR, et al. A phase III randomized trial of BAY 12-9566 (tanomastat) as maintenance therapy in patients with advanced ovarian cancer responsive to primary surgery and paclitaxel/ platinum containing chemotherapy: a NCI of Canada Clinical Trials Group Study. Gynecol Oncol. 2006;102(2):300-308.

9. Pfisterer J, Weber B, Reuss A, et al. Randomized phase III trial of topotecan following carboplatin and paclitaxel in first-line treatment of advance d ovarian cancer: a gynecologic cancer intergroup trial of the AGO-OVAR and GINECO. J Natl Cancer Inst. 2006;98(15): 1036-1045.

10. Cure H, Battista C, Guastalla JP, Fabbro M, Tubiana N, Bourgeouis H, et al. Phase III randomized trial of high-dose chemotherapy (HDC) and peripheral blood stem cell (PBSC) support as consolidation in patients with advanced ovarian cancer: 5-year follow up of a GINECO/FNCLCC/ SFGM-TC study. ASCO Annual Meeting Proceedings, 2004.

11. Hall GD, Brown JM, Coleman RE, et al. Maintenance treatment with interferon for advanced ovarian cancer: results of the Northern and Yorkshire gynaecology group randomized phase III study. Br J Cancer. 2004;91(4):621-626. 
12. Berek JS, Taylor PT, Gordon A, et al. Randomized, placebo controlled study of oregovomab for consolidation of clinical remission in patients with advanced ovarian cancer. J Clin Oncol. 2004;22(17): 3507-3516.

13. Berek J, Taylor P, McGuire W, Smith LM, Schultes B, Nicodemus CF. Oregovomab maintenance monoimmunotherapy does not improve outcomes in advanced ovarian cancer. J Clin Oncol. 2009;27(3):418-425.

14. Sorbe B. Consolidation treatment of advanced ovarian carcinoma with radiotherapy after induction chemotherapy. Int J Gynecol Cancer. 2003;13:Suppl 2:181-185.

15. Varia MA, Stehman FB, Bundy BN, et al. Intraperitoneal radioactive phosphorus $\left({ }^{32} \mathrm{P}\right)$ versus observation after negative second-look laparotomy for stage III ovarian carcinoma: a randomized trial of the Gynecologic Oncology Group. J Clin Oncol. 2003;21(15):2849-2855.

16. Conte PF, Favilli G, Gadducci A, et al. Final result results of after-6 protocol 1: a phase III trial of observation versus 6 courses of paclitaxel in advanced ovarian cancer patients in complete response after platinum-paclitaxel chemotherapy. [abstract]. J Clin Oncol. 2007;25(18s):275s.

17. Markman M, Liu PY, Wilczynski S, et al. Phase III randomized trial of 12 versus 3 months of maintenance paclitaxel in patients with advanced ovarian cancer after complete response to platinum and paclitaxelbased chemotherapy: A Southwest Oncology Group and Gynecologic Oncology Group Trial. J Clin Oncol. 2003;21:2460-2465.

18. Markman M, Liu PY, Moon J, et al. Impact on survival of 12 versus 3 monthly cycles of paclitaxel $\left(175 \mathrm{mg} / \mathrm{m}^{2}\right)$ administered to patients with advanced ovarian cancer who attained a complete response to primary platinum-paclitaxel: follow-up of a Southwest Oncology Group and Gynecologic Oncology Group phase 3 trial. Gynecol Oncol. 2009;14(2):195-198.

19. Rowinsky EK, Donehower RC. Paclitaxel (taxol). N Engl J Med. 1995; 332(15):1004-1014.

20. Ozols RF. Maintenance therapy in advanced ovarian cancer: progressionfree survival and clinical benefit. J Clin Oncol. 2003;21(13):2451-2453.

21. Herzog TJ, Coleman RL, Markman M, Cella D, Thigpen JT. The role of maintenance therapy and novel taxanes in ovarian cancer. Gynecol Oncol. 2006;102:218-225.
22. Singer JW, Shaffer S, Baker B, et al. Paclitaxel poliglumex (XYOTAX; CT-2103); an intracellularly targeted taxane. Anticancer Drugs. 2005; 16(3):243-254.

23. Paclitaxel or polyglutamate paclitaxel or observation in treating patients with stage III or stage IV ovarian epithelial or peritoneal cancer. Clinical Trials.gov; 2009.

24. Mabuchi S, Terai Y, Morishige K, et al. Maintenance treatment with bevacizumab prolongs survival in an in vivo ovarian cancer model. Clin Cancer Res. 2008;14(23):7781-7789.

25. Carboplatin and paclitaxel with or without bevacizumab in treating patients with stage III or stage IV ovarian epithelial, primary peritoneal cancer, or fallopian tube cancer. Clinicaltrials.gov; 2009.

26. A study of carboplatin and gemcitabine plus bevacizumab in patients with ovary, peritoneal, or fallopian tube carcinoma. Clinical Trials. gov; 2009.

27. Efficacy and safety of pazopanib monotherapy after first line chemotherapy in ovarian, fallopian tube, or primary peritoneal cancer. Clinicaltrials.gov; 2009.

28. Carboplatin and paclitaxel with or without cediranib in treating women with relapsed ovarian epithelial cancer, fallopian tube cancer, or primary peritoneal cancer. Clinical Trials.gov; 2009

29. Monoclonal antibody vaccine therapy in treating patients with ovarian epithelial, fallopian tube, or peritoneal cancer. Clinicaltrials. gov; 2009.

30. Robinson WR, Davis N, Rogers AS. Paclitaxel maintenance chemotherapy following intraperitoneal chemotherapy for ovarian cancer. Int J Gynecol Cancer. 2008;18(5):891-895.

31. Vasey PA, Jayson GC, Gordon A, et al. Phase III randomized trial of doecetaxel-carboplatin versus paclitaxel-carboplatin as first-line chemotherapy for ovarian carcinoma. J Natl Cancer Inst. 2004;96(22): 1682-1691.

32. Goodin S, Kane MP, Rubin EH. Epithilones: mechanism of action and biologic activity. J Clin Oncol. 2004;22 (10):2015-2025.

33. Ibrahim NK, et al. Phase I and pharmacokinetic study of ABI-007, a Crmophor free, protein-stabilized, nanoparticle formulation of paclitaxel. Clin Cancer Res. 2002;8(5):1038-1344.

34. NIH. Clinicaltrials.gov; 2009.
International Journal of Women's Health

\section{Publish your work in this journal}

The International Journal of Women's Health is an international, peerreviewed open-access journal publishing original research, reports, reviews and commentaries on all aspects of women's healthcare including gynaecology, obstetrics, and breast cancer. Subject areas include: Chronic conditions (migraine headaches, arthritis, osteoporosis);

\section{Dovepress}

Endocrine and autoimmune syndromes; Sexual and reproductive health; Psychological and psychosocial conditions. The manuscript management system is completely online and includes a very quick and fair peer-review system. Visit http://www.dovepress.com/ testimonials.php to read real quotes from published authors. 